\title{
Experimental inflammation following dural application of complete Freund's adjuvant or inflammatory soup does not alter brain and trigeminal microvascular passage
}

\author{
Cornelia Lundblad', Kristian A. Haanes², Gustaf Grände ${ }^{1}$ and Lars Edvinsson ${ }^{1,2^{*}}$
}

\begin{abstract}
Background: Migraine is a paroxysmal, disabling primary headache that affects $16 \%$ of the adult population. In spite of decades of intense research, the origin and the pathophysiology mechanisms involved are still not fully known. Although triptans and gepants provide effective relief from acute migraine for many patients, their site of action remains unidentified. It has been suggested that during migraine attacks the leakiness of the blood-brain barrier (BBB) is altered, increasing the passage of anti-migraine drugs. This study aimed to investigate the effect of experimental inflammation, following dural application of complete Freund's adjuvant (CFA) or inflammatory soup (IS) on brain and trigeminal microvascular passage.
\end{abstract}

Methods: In order to address this issue, we induced local inflammation in male Sprague-Dawley-rats dura mater by the addition of CFA or IS directly on the dural surface. Following 2, 24 or $48 \mathrm{~h}$ of inflammation we calculated permeability-surface area product (PS) for $\left[{ }^{51} \mathrm{Cr}\right]$-EDTA in the trigeminal ganglion (TG), spinal trigeminal nucleus, cortex, periaqueductal grey and cerebellum.

Results: We observed that $\left.{ }^{51} \mathrm{Cr}\right]$-EDTA did not pass into the central nervous system (CNS) in a major way. However, $\left[{ }^{51} \mathrm{Cr}\right]$-EDTA readily passed the TG by $>30$ times compared to the CNS. Application of CFA or IS did not show altered transfer constants.

Conclusions: With these experiments we show that dural IS/CFA triggered TG inflammation, did not increase the BBB passage, and that the TG is readily exposed to circulating molecules. The TG could provide a site of anti-migraine drug interaction with effect on the trigeminal system.

Keywords: Microvascular passage, Transfer constant, Trigeminal ganglion, Inflammatory soup, Complete Freund's Adjuvant

\section{Background}

Migraine is a neurological disorder that afflicts up to $16 \%$ of the adult population in the Western countries [1]. It is episodic, often with moderate to severe disabling headache, associated with sensory and autonomic symptoms, phonophobia and photophobia, and cognitive symptoms. Many researchers consider migraine to be a

\footnotetext{
* Correspondence: Lars.Edvinsson@med.lu.se

'Department of Medicine, Institute of Clinical Sciences, University Hospital, Lund University, 22185 Lund, Sweden

${ }^{2}$ Department of Clinical Experimental Research, Copenhagen University Hospital, Glostrup, Denmark
}

disorder in which Central Nervous System (CNS) dysfunction plays a pivotal role while various parts of the trigeminovascular system are necessary for the expression of peripheral symptoms and aspects of pain [2].

Several drugs have been shown to reduce migraine symptoms, or elicits migraine-like attacks [3]. Studies, both in man and in experimental animals, have revealed anti-migraine effects of triptans, gepants (calcitonin gene-related peptide (CGRP) antagonists) and CGRP antibodies [4-7]. However, due to the size and the pharmacokinetics of these drugs it is suggested that only a modest passage may occur across the blood-brain 
barrier (BBB) [8]. The published figures on BBB passage are in the vicinity of $3 \%$ when given at clinically effective doses of triptans/gepants [5, 9-11]. While these molecules have a small size, the CGRP antibodies will not likely cross the $\mathrm{BBB}$ at all and therefore unlikely to antagonize receptor sites within the CNS [5].

In order to neutralize this argument some researchers have suggested that the $\mathrm{BBB}$ is modified during migraine attacks and may show enhanced passage [12]. From a purely mechanistic viewpoint the BBB consists of a myriad of molecules, including metalloproteases, as well as claudins and integrins which would negate a dynamic opening of the closed tight junctions which are an essential part of the BBB $[13,14]$. Another aspect that must be considered, that has not been proven, is modification of transport guided passage across the BBB which could be modified during the attacks.

Therefore, it is possible that candidates for targets of migraine treatment may reside outside the $\mathrm{BBB}$; the dural vasculature, the trigeminal ganglion (TG) and in CNS regions lacking a BBB such as pituitary and pineal glands [15] and at nerve afferents that connect to the TG. The dural vasculature, including the middle meningeal artery (MMA) has long been considered as a target based on the vasogenic theory of migraine $[16,17]$. We and others have shown that both sumatriptan and telcagepant can inhibit CGRP induced vasodilation in the human MMA, illustrating one of its potential targets $[18,19]$. However, not all vasodilators cause migraine and triptans are not effective in all migraine patients [20].

The other possibility is the activation and involvement of the TG with its afferents which innervates the dura mater and the MMA [21]. Based on preceding studies on TG cell [22] and TG organ cultures [23], we have recently shown that the addition of complete Freund's adjuvant (CFA) or inflammatory soup (IS) on the dura mater causes an increase in phosphorylated extracellular signal-regulated kinases (p-ERK1/2) activation and interleukin (IL)-1 $\beta$ expression in the ipsilateral TG [24]. The application of inflammatory substances on the dura mater, in addition to chemical stimulation of the dural receptive fields, cause hypersensitivity to mechanical and thermal stimulation, as well as activation in the TG and in brain stem trigeminal neurons, similar to what could be expected in migraine $[17,24]$. BBB permeability has never been investigated using this model. It is highly relevant to ask this question as it has been suggested that the BBB might change during a migraine attack in effort to explain how anti-migraine drugs can reach the CNS $[12,25-28]$. In addition, it is relevant to compare the permeability to the TG and evaluate any potential alterations.

Here we report that there are no differences in the permeability into the CNS after experimental inflammation and there are no significant changes in brain water content or in permeability. Interestingly, we show that the TG is more than 30 times more permeable than the CNS at normal conditions and thereby supporting the idea that anti-migraine drugs may reach much higher and clinically relevant levels within the TG than they do in the CNS.

\section{Methods}

\section{Animals}

The experimental protocol was approved by the local Ethical Committee for Animal Research (M38-13). Adult male Sprague-Dawley rats $(n=35)$ (Taconic Copenhagen, Denmark) were used to investigate the brain water content and the permeability-surface area product (PS) for $\left[{ }^{51} \mathrm{Cr}\right]$-EDTA. There was a reversed $12-12$ light cycle, with darkness during the day. The animals were treated in accordance with the national guidelines for laboratory animals (publications DFS 2004:4, the Swedish Board of Agriculture)

The animals were anesthetized by intraperitoneal injection of sodium pentobarbital $(60 \mathrm{mg} / \mathrm{kg}$, Pentobarbitalnatrium, vet.APL, $60 \mathrm{mg} / \mathrm{ml}$, APL, Stockholm) and then placed on a heating pad and in a stereotactic head holder. Body core temperature was kept at $37{ }^{\circ} \mathrm{C}$ by a feedback circuit controlled by continuous measurement of rectal temperature. A parasagittal craniotomy, with a diameter of $6 \mathrm{~mm}$, was performed in the left parietal bone between the coronal and lambdoid sutures with the center approximately $5 \mathrm{~mm}$ from the coronal suture and $5 \mathrm{~mm}$ from the sagittal suture, after a scalp incision. The animals were randomly assigned to three different solutions applied to the surface of the exposed dura in a volume of $10 \mu \mathrm{L}$; i) vehicle (saline), ii) CFA used as stock solution (Sigma Aldrich, Life Science, US), and iii) an IS. The recipe of IS was adapted from Strassman and colleagues [29] and contains $10 \mu \mathrm{M}$ each of bradykinin, serotonin and prostaglandin E2, $100 \mu \mathrm{M}$ histamine at a $\mathrm{pH}$ of 5.0 (all obtained from Sigma Aldrich). After 15 min CFA or IS was removed by flushing with saline. Surgical staples were used to close the scalp incision and the animals were moved to another heating pad for recovering. At 2, 24 or $48 \mathrm{~h}$ following application to the dura, the animals were re-anesthetized. Anesthesia was induced by placing the rat in a covered glass-container with a continuous supply of isoflurane (Isofluran Baxter, Baxter Medical, Stockholm, Sweden). After tracheostomy, the rat was connected to a ventilator and anesthesia was maintained by inhalation of 1.4-1.7\% of isoflurane in room air. End tidal $\mathrm{pCO}_{2}$ was monitored continuously with a capnograph (CapnoTrue AMP, bluepoint MEDICAl, GmbH, Selmsdorf, Germany). The left femoral artery was canulated for measurement of mean arterial blood pressure (MAP) and to obtain arterial blood samples used for measurement of the physiological 
Table 1 Physiological parameters in animals where Ki for ${ }^{51} \mathrm{Cr}$-EDTA was determined. Hematocrit in animals treated with CFA was lower than in the vehicle group at $24 \mathrm{~h}$ following application to the dura. No other differences regarding measured physiological data were observed between the groups

\begin{tabular}{|c|c|c|c|c|c|c|}
\hline & Vehicle $(n=6)$ & CFA 2 h $(n=6)$ & CFA 24 h $(n=5)$ & CFA 48 h $(n=5)$ & IS $2 \mathrm{~h}(n=6)$ & IS $24 \mathrm{~h}(n=4)$ \\
\hline MAP $(\mathrm{mmHg})$ & $98 \pm 7$ & $88 \pm 3$ & $87 \pm 6$ & $105 \pm 7$ & $85 \pm 5$ & $104 \pm 9$ \\
\hline Hct (\%) & $50 \pm 2$ & $46 \pm 3$ & $38 \pm 2^{*}$ & $42 \pm 1$ & $46 \pm 2$ & $42 \pm 2$ \\
\hline $\mathrm{pCO}_{2}(\mathrm{kPa})$ & $4.6 \pm 0.1$ & $4.6 \pm 0.1$ & $4.7 \pm 0.2$ & $4.5 \pm 0.1$ & $4.6 \pm 0.1$ & $4.8 \pm 0.2$ \\
\hline $\mathrm{pO}_{2}(\mathrm{kPa})$ & $11.5 \pm 0.1$ & $11.6 \pm 0.1$ & $11.0 \pm 0.2$ & $12.7 \pm 0.5$ & $11.4 \pm 0.3$ & $11.4 \pm 0.5$ \\
\hline $\mathrm{Na}^{+}(\mathrm{mmol})$ & $136.3 \pm 0.6$ & $138.0 \pm 1.6$ & $138.6 \pm 1.0$ & $137.0 \pm 0.5$ & $137.0 \pm 0.5$ & $136.5 \pm 0.9$ \\
\hline $\mathrm{K}^{+}(\mathrm{mmol})$ & $4.82 \pm 0.39$ & $4.65 \pm 0.39$ & $4.36 \pm 0.13$ & $4.40 \pm 0.13$ & $4.57 \pm 0.14$ & $4.55 \pm 0.22$ \\
\hline $\mathrm{pH}$ & $7.50 \pm 0.02$ & $7.45 \pm 0.02$ & $7.46 \pm 0.02$ & $7.46 \pm 0.01$ & $7.48 \pm 0.01$ & $7.48 \pm 0.01$ \\
\hline
\end{tabular}

Differences in plasma volumes in tissue was found following application of both CFA and IS compared to vehicle $\left({ }^{*} p<0.05\right)$

parameters $\mathrm{pH}, \mathrm{pCO}_{2}, \mathrm{pO}_{2}$, hematocrit and electrolytes. The left femoral vein was canulated and used for infusions.

\section{Tissue Water Content and the permeability-surface area product (PS) for $\left[{ }^{51} \mathrm{Cr}\right]$-EDTA}

Change in microvascular permeability and surface area for transvascular exchange following the various treatments was investigated by measurement of the permeabilitysurface area product (PS) for $\left[{ }^{51} \mathrm{Cr}\right]$-EDTA, as described earlier [30, 31]. For this purpose the animals received a bolus infusion of about $370 \mathrm{kBq}$ of the tracer $\left[{ }^{51} \mathrm{Cr}\right]-$ EDTA, (0.5 mL) (GE Health Care, Stockholm, Sweden). The bolus infusion was followed by a continuous infusion of the tracer at a rate of $0.33 \mathrm{~mL} / \mathrm{h}(3.7 \mathrm{MBq} / \mathrm{mL})$. Arterial blood samples $(10 \mu \mathrm{L})$ for analyses of plasma $\left[{ }^{51} \mathrm{Cr}\right]$-EDTA concentration were collected at 2.5, 5, 10, 15, 25, 35 and $40 \mathrm{~min}$ post start of the bolus injection. After $37 \mathrm{~min}$, a bolus dose of about $25 \mathrm{kBq}$ of [ $\left.{ }^{125} \mathrm{I}\right]$-albumin dissolved in $0.1 \mathrm{~mL}$ isotonic saline was given in the femoral vein for

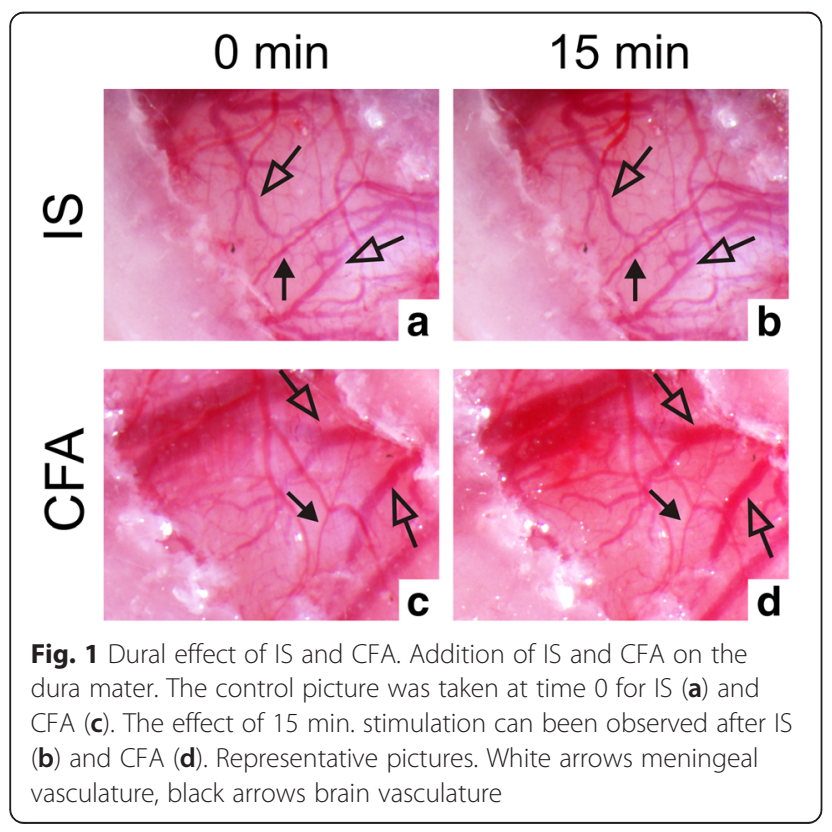

calculation of plasma volume in the brain. Three min later, the experiment was finished by an arterial blood sample and the animal was sacrificed by decapitation. Before each experiment $\left[{ }^{125} \mathrm{I}\right]$-albumin was purified from free iodine, using centrifugal filtration. The brain and the TGs were removed and put on a chilled support. In order to analyze PS in the cortex, periaqueductal gray (PAG), trigeminal nuclei caudalis (TNC) and cerebellum, tissue structures were extracted and immediately weighed. Tissue and blood tracer activities of $51 \mathrm{Cr}$-EDTA and ${ }^{125} \mathrm{I}$-albumin were determined in a gamma counter. Tissue samples were then dried in an oven for $24 \mathrm{~h}$ at $100{ }^{\circ} \mathrm{C}$ and brain water content was calculated as [(wet weight-dry weight)/ wet weight) $] \times 100$. Arterial hematocrit was measured before and during tracer infusion in order to convert blood concentrations into plasma concentrations. The blood to brain transfer constant $\left(\mathrm{K}_{\mathrm{i}}\right)$ for $\left[{ }^{51} \mathrm{Cr}\right]$-EDTA was then calculated according to the following equation $K_{i}=B /{ }_{0} \int^{T} C_{a}(t) d t$ [32], where $B$ is the amount of tracer that has moved from blood to brain (tissue uptake of tracer minus regional tracer concentration in plasma), $C_{a}$ is concentration of the tracer in arterial plasma as a function of time, and $\mathrm{T}$ is the duration of the experiment. $\mathrm{K}_{\mathrm{i}}$ is a function of capillary plasma flow per unit mass of tissue (FV) and the permeabilitysurface area product (PS), the latter reflecting microvascular permeability and surface area available for diffusional exchange. The mathematical expression for this relationship is $K_{i}=F V\left[1-e^{-P S / F V}\right]$ which can be rewritten as PS $=-\mathrm{FV} \ln \left(1-\mathrm{K}_{\mathrm{i}} / \mathrm{FV}\right)$ [33]. From this expression it can be deduced that with a $\mathrm{K}_{\mathrm{i}} / \mathrm{FV}$ ratio of less than 0.1 the $K_{i}$ value approximates PS with an error of less than $6 \%$ [34].

\section{Evans blue}

The method has recently been published; briefly $2 \% \mathrm{Ev}$ ans blue in saline was injected in the tail vein $(4 \mathrm{ml} / \mathrm{kg}$ ) during anaesthesia (Eftekhari et al 2015). After $30 \mathrm{~min}$ the animal was sacrificed and the tissues dissected out. Evans blue couple to circulating albumin and forms a 
Table 2 Plasma volume in tissue in analyzed brain structures. Plasma volume in analyzed structures following application of CFA or IS to the dura was compared to plasma volume in analyzed structure following application of vehicle

\begin{tabular}{|c|c|c|c|c|c|c|}
\hline & Control & CFA $2 \mathrm{~h}$ & CFA $24 \mathrm{~h}$ & CFA $48 \mathrm{~h}$ & $\mathrm{IS} 2 \mathrm{~h}$ & IS $24 \mathrm{~h}$ \\
\hline Cortex $(\mu \mathrm{L} / \mathrm{mg})$ & $7.3+0.6$ & $12.2 \pm 2.5$ & $12.6 \pm 0.8^{*}$ & $14.6+3.0^{*}$ & $11.3 \pm 0.7^{*}$ & $11.0 \pm 2.0$ \\
\hline PAG $(\mu \mathrm{L} / \mathrm{mg})$ & $6.0 \pm 0.6$ & $15.3 \pm 3.2^{*}$ & $12.8 \pm 1.4^{*}$ & $8.8 \pm 1.5$ & $13.8 \pm 2.9$ & $11.8 \pm 2.3$ \\
\hline Cerebellum ( $\mu \mathrm{L} / \mathrm{mg})$ & $9.5 \pm 0.4$ & $14.2 \pm 0.8^{*}$ & $12.8 \pm 0.5$ & $29.4 \pm 6.2 *$ & $13.8 \pm 1.7$ & $16.3 \pm 1.3^{*}$ \\
\hline L Spinal Trigeminal nucleus ( $\mu \mathrm{L} / \mathrm{mg})$ & $10.3 \pm 1.7$ & $14.3 \pm 2.3$ & $21.2 \pm 2.1^{*}$ & $44.0 \pm 14.4^{*}$ & $12.5 \pm 3.5^{*}$ & $27.0 \pm 8.9$ \\
\hline R Spinal Trigeminal nucleus ( $\mu \mathrm{L} / \mathrm{mg})$ & $12.8 \pm 2.6$ & $13.7 \pm 1.6$ & $19.4 \pm 1.8$ & $23.6 \pm 3.4^{*}$ & $16.0 \pm 1.8$ & $28.0 \pm 11.0$ \\
\hline L Trigeminal Ganglion $(\mu \mathrm{L} / \mathrm{mg})$ & $52.3 \pm 4.3$ & $53.3 \pm 12.5$ & $48.0 \pm 8.0$ & $25.8 \pm 3.1$ & $40.5 \pm 13.4$ & $59.3 \pm 10.3$ \\
\hline R Trigeminal Ganglion $(\mu \mathrm{L} / \mathrm{min} / \mathrm{g})$ & $54.5 \pm 5.7$ & $51.7 \pm 10.4$ & $68.2 \pm 13.9$ & $22.0 \pm 2.6$ & $56.7 \pm 15.5$ & $83.8 \pm 27.4$ \\
\hline
\end{tabular}

Differences in plasma volumes in tissue was found following application of both CFA and IS compared to vehicle ( ${ }^{*} p<0.05$ )

large complex (EBA) which does not pass the BBB at the resting state.

\section{Photo microscopy}

Sprague-Dawley rats $(n=3)$ were anesthetized and IS or CFA was added (see above) and monitored with a photo microscope (Olympus SZX10) at baseline and after 15 min following the addition of the inflammatory substances. For the three rats, parasagittal craniotomy, with a diameter of $6 \mathrm{~mm}$, was performed and analyzed on both the left and right parietal bone.

\section{Statistical analysis}

All data are expressed as mean $\pm \mathrm{SE}$, where $\mathrm{n}$ equals individual animals. Data were analyzed statistically using Prism 6 (GraphPad Software, Inc, CA). Differences between groups were tested using non-parametric analyzes of variance with Kruskal-Wallis test followed by Dunn's test for multiple comparisons. Each group was compared with the vehicle group.

\section{Results}

\section{Physiological data}

The physiological parameters were examined after 2, 24 and $48 \mathrm{~h}$ for CFA, and at 2 and $24 \mathrm{~h}$ for IS (Table 1). No significant differences regarding measured physiological data $\left(\mathrm{Na}^{+}, \mathrm{K}^{+}, \mathrm{pH}, \mathrm{pCO}_{2}, \mathrm{pO}_{2}\right.$ and $\left.\mathrm{MAP}\right)$ were observed between the groups.

\section{Photo microscopy}

We were interested in observing whether the addition of IS or CFA in our model had any direct effect at the place of addition on the dura mater, using photo microscopy. It is worth pointing out that removal of the bone for the direct application on the dura, reduces the possible changes that can be observed in meningeal vasculature (see discussion). 15 min after the addition of IS (Fig. 1a/b) and CFA (Fig. 1c/d) we did not observe any changes in the meningeal vasculature (white arrows). However, there appears to be an weak increase in brain blood vessel diameter (black arrows), particularly after CFA.

\section{Permeability-surface area product for $\left[{ }^{51} \mathrm{Cr}\right]$ - EDTA}

In order to study the permeability surface area product (PS), $\left[{ }^{51} \mathrm{Cr}\right]$-EDTA was injected as a bolus dose (370 bq) followed by continuous infusion $0.33 \mathrm{ml} / \mathrm{h}(3.7 \mathrm{MBq} /$ $\mathrm{ml})$. To correct for tissue plasma volume, a bolus dose of $\left[{ }^{125} \mathrm{I}\right]$-albumin was injected $3 \mathrm{~min}$ before the experiment was terminated and tissue collected. The plasma content, which is used to correct the $\left[{ }^{51} \mathrm{Cr}\right]$-EDTA data (Table 2), was significantly higher $2 \mathrm{~h}$ after CFA treatment in PAG and cerebellum. $48 \mathrm{~h}$ after CFA treatment the plasma content was significantly higher in the spinal trigeminal nucleus. For IS a small increase was observed in the cerebellum at $24 \mathrm{~h}$ and with a minor change in the TNC. This increased plasma content suggests a larger blood volume that was increased due to the inflammation.

We investigated the left and right TG and TNC individually. IS and CFA was only added on the left parietal bone, and we were interested in observing a direct link between the dural application and the corresponding TG and TNC structures. The control data where saline has been applied to the dura mater show that the TG is $>30$ times more permeable than cerebellum/cortex and $>200$ times than the PAG (Fig. 2a). In addition, the permeability of the TG was also $>30$ times higher than the spinal TNC. This clearly illustrates that the TG has much higher permeability than the CNS and is also in agreement with the $3 \%$ of triptans/gepants that can reach the CNS [5, 9-11] as the cortex/cerebellum has a permeability that is $\sim 3 \%$ of TG. In addition we also show that Evans blue, that cannot pass the BBB (Evans blue binds to serum albumin, creating a large molecule that can be visualized), colours the TG but not the brain (Fig. 2b). The dura mater was likewise "blue" after the administration of EBA (Fig. 2c). Due to the operation and manipulations we did not quantify the dura mater permeability.

We further characterized the changes in permeability following the addition of CFA and IS onto the dura. There were no significant increases in the permeability of the cortex, cerebellum or PAG following either of the inflammation triggers (Fig. 3) compared to vehicle, 


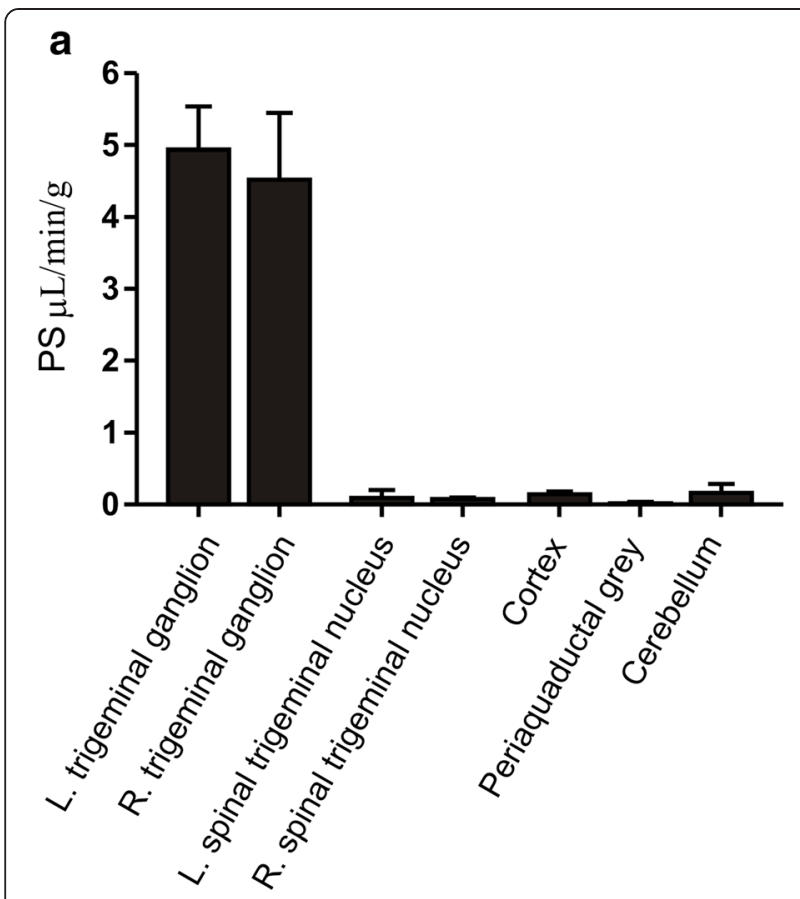

b

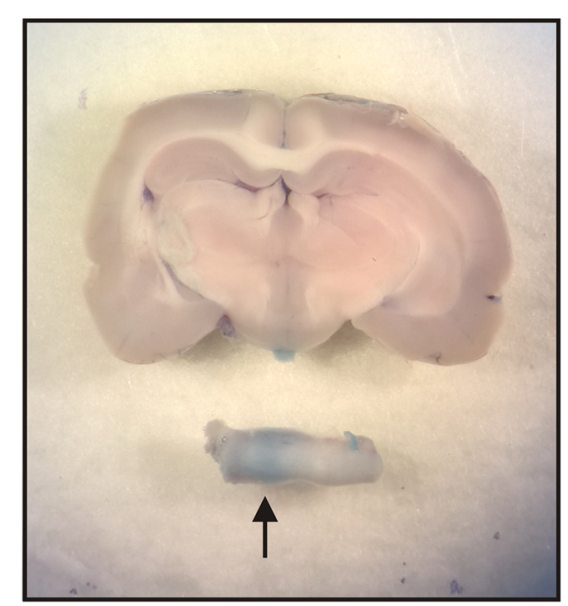

C

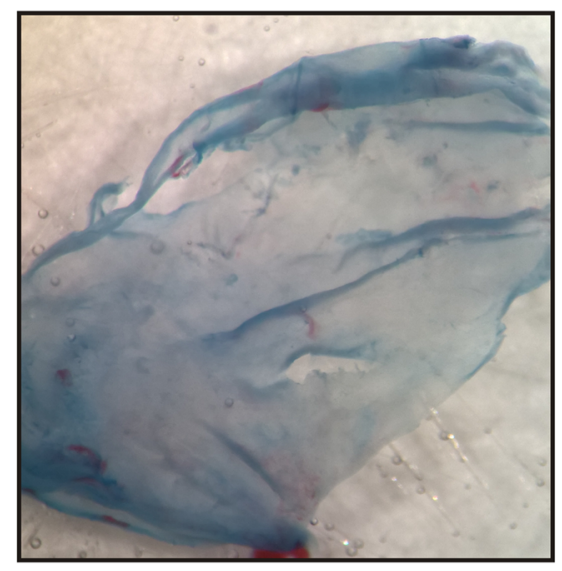

Fig. 2 Comparison of the PS for the different structures analysed. a The PS of the TG is much larger than that of the other structures investigated. $\mathbf{b}$ Rat brain and TG after perfusion of Evans blue shows that the TG is permeable whereas the brain is not. c The dura mater is permeable to Evans blue

suggesting that permeability does not change in the inflammatory models. A small permeability increase in the spinal TNC was observed after $24 \mathrm{~h}$ of CFA, although the change is nearly negligible as it after CFA still is $>10$ times less permeable than the TG (Fig. 4). We observed a small decrease in the left TG with the same tendency in the right TG, which could be due to the increased ganglion activation. We did not observe any significant changes in any of the structures examined after either 2 or $24 \mathrm{~h}$ after the addition of IS.

To ensure that there was no oedema in the brain, we measured the brain water content (Additional file 1: Figure S1). There were no significant changes in water content in the CNS. Only the left TG showed an increase of $10 \%$ at $48 \mathrm{~h}$ after CFA application onto the dura. This also supports the permeability studies as a lowered permeability in the BBB, most likely would have led to increased water content.

\section{Discussion}

This is the first study designed to quantify and compare the permeability in the TG and the CNS. We present results that show the PS (and hence the permeability) was more than 30 times higher in the TG than in other structures (CNS) analysed, strongly indicating that the TG is indeed outside the BBB. The addition of the inflammatory agents, IS or CFA, did not change the permeability in the CNS structures, despite demonstrating an inflammatory reaction in the TG [24]. This will be discussed below in the relation to the pharmacokinetics/ $\mathrm{BBB}$ and the TG as a possible target for acute migraine treatment. The dura mater does not have a barrier to EBA (Fig. 2c), since we operated in this area it was not included in the quantification. It is worth noting that it is the part of TG that contains the neurons that are "blue" in the TG and that the pale part is the trigeminal nerves (Fig. 2b).

\section{Changes in plasma content}

Tissue plasma volume was determined by measured radioactivity of ${ }^{125} \mathrm{I}$, which represents radioactivity from only plasma. Leakage of infused radioactive albumin during the $3 \mathrm{~min}$ of its circulation time must be regarded as insignificant, and has been analysed before [35]. This means that the calculated increase in plasma volume, most likely, was an effect of inflammatory-induced vasodilation rather than extravasation. In addition, we observed no changes in brain water content which illustrates 


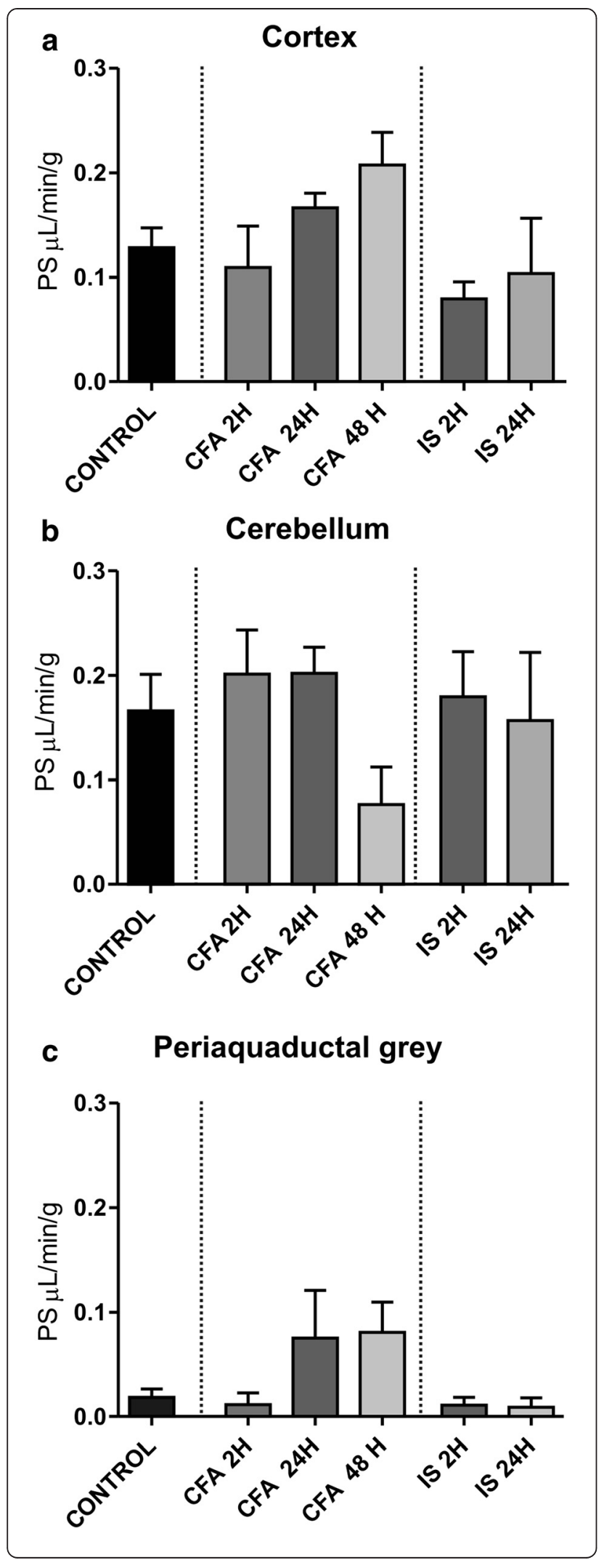

Fig. 3 PS in the cortex cerebellum and PAG after treatment with CFA or IS. The figure shows PS for ${ }^{51} \mathrm{Cr}$-EDTA in the cortex (a), cerebellum (b) and periaquaductal grey (c). PS for treatment following application of CFA or IS to the dura was compared to PS following application of vehicle to the dura. ${ }^{*} p \leq 0.05$

that there is not a large extravasation of water and that the intracranial pressure is maintained after the addition of either of the inflammatory substances. It is worth noting that an increase in plasma volumes after application of CFA and IS may affect the calculations of PS, especially in areas with low permeability for $\left[{ }^{51} \mathrm{Cr}\right]$-EDTA in terms of in underestimation of PS (Ki), as B is indirectly dependent on plasma volume (see Methods), The theory behind the present method has been discussed before, see Chodobski and colleagues [14].

\section{Permeability of TG/CNS in relation to BBB}

The results presented here, show that permeability was significantly higher in the TG than in other structures analysed (Fig. 2), both after addition of vehicle and after application of CFA or IS. This illustrates that the drugavailability will be much higher in the TG compared to the CNS and supports this as a potential target for antimigraine drugs [9]. The inflammatory model has been utilized in several studies and show similar findings to migraine for example TG activation with an increase in p-ERK1/2 activation and IL-1 $\beta$ expression, hypersensitivity to mechanical and thermal stimulation, as well as activation in the TG and in the brain stem trigeminal neurons, similar to what could be expected in migraine $[17,24,29,36,37]$. Since it has been suggested that the $\mathrm{BBB}$ in migraine patients might be defective or transiently altered [12], we tested if the BBB was altered in the present method. Obviously this is not a "true" migraine model; it may be regarded as a surrogate method. IS has been used in many studies for the understanding of for example trigeminal involved sensitization [17]. CFA is a well-known method to induce local inflammation and we postulate that this may share aspects with the more chronic forms of migraine. In relation to other models, there exists one study on repeated cortical spreading depression and the $\mathrm{BBB}$ in rats [25]. Here a mild $\mathrm{BBB}$ breakdown/leakage was observed including brain edema and protein leakage. However, the cortical depression has to be very strong for these effects, for example after cerebral ischemia where there is a strong cortical spreading depression, it still takes several hours for the BBB to be broken down [38]. In addition, there are no clear proof of breakdown or leakage in the $\mathrm{BBB}$ in patients during migraine attacks [8]

Interestingly, in a study by Markowitz and colleagues, extravasated ${ }^{125}$ I-BSA or Evans blue was noted in the dura of saline-perfused rats following intravenous 


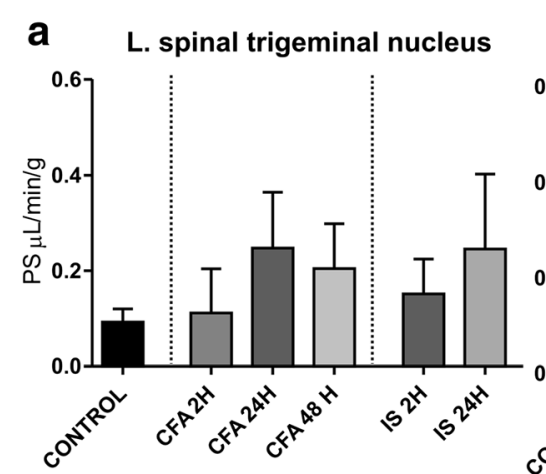

b R. Spinal trigeminal nucleus

C L. Trigeminal ganglion
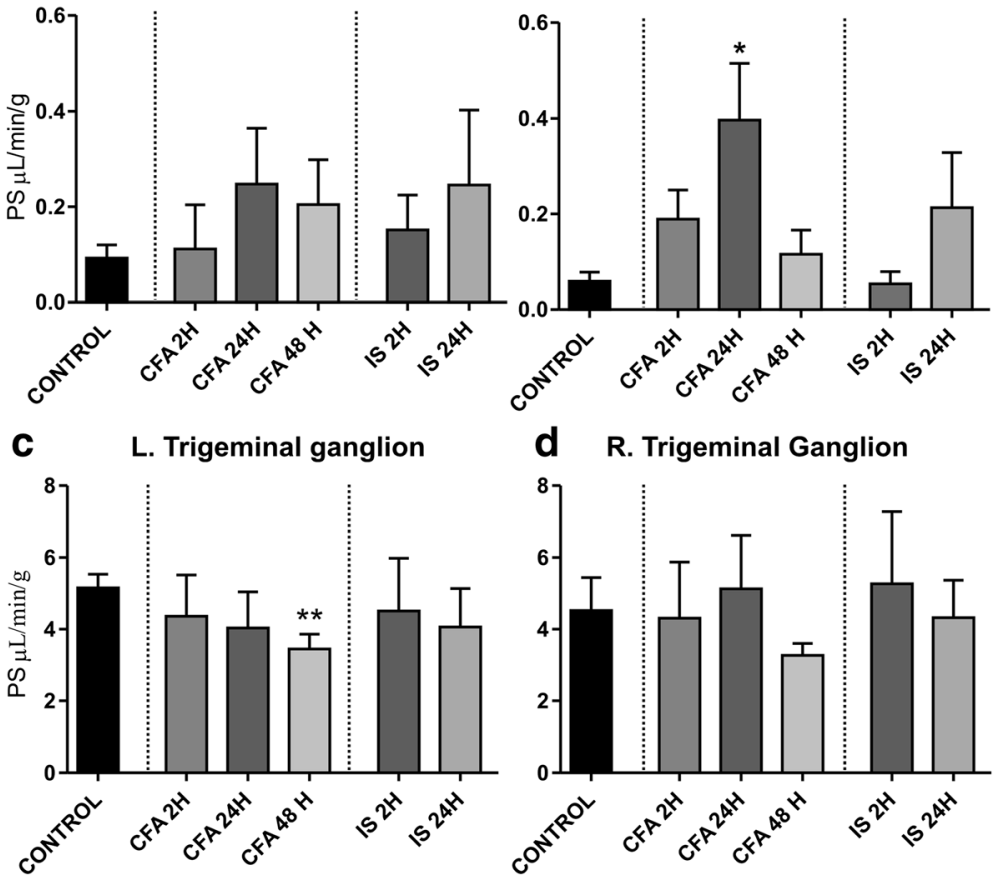

d R. Trigeminal Ganglion

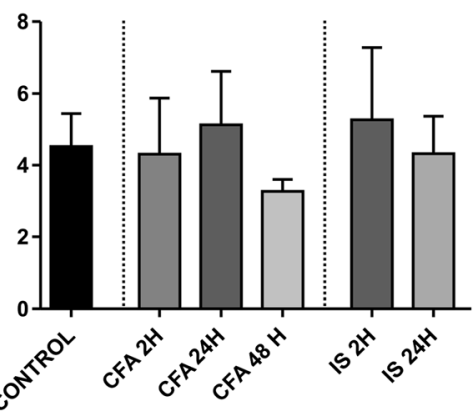

Fig. 4 PS in the TG and spinal trigeminal nucleus after treatment with CFA or IS. The figure shows PS for ${ }^{51} \mathrm{Cr}$-EDTA in the left TNC (a), right TNC (b), left TG (c), and right TG (d). PS for treatment following application of CFA or IS to the dura was compared to PS following application of vehicle to the dura. ${ }^{*} p \leq 0.05$

capsaicin or following electrical stimulation of the rat TG. Extravasation was however not observed in the brain following chemical, electrical, or immunological stimulation nor following topical application of capsaicin to the pial surface or injection into the cortex of rats. The data are therefore showing similar indication as us, with our inflammatory model [39]. As we did not observe any alteration in the $\mathrm{BBB}$, we add our data to the negative experiments that the $\mathrm{BBB}$ does not change its permeability during migraine attacks. Our results are in agreement with clinical data observations [26-28].

\section{Direct effects on vasculature upon the addition of CFA and IS}

The addition of the inflammatory substances onto the dura mater raises an interesting question in relation to the vasogenic theory. Does the inflammatory substance lead to vasodilation? IS caused a mild contraction and CFA had no vasomotor effect per se on the in vitro MMA, studied using wire myography [24]. In our model, we could however not observe any changes in the meningeal arteries. Interestingly, Williamson and colleagues showed that in closed cranial window an increase in the MMA diameter of up to $180 \%$ could be seen with the addition of CGRP [40]. However, in open cranial window only $15 \%$ was observed [41], and therefore changes are difficult to detect. The effect of IS and CFA on the MMA will require further analysis, but this is not in the scope of this study. Here we show that in our model of application of IS and CFA we do not see any changes in MMA, we do however see an increased blood flow to this area, particularly for CFA.

\section{Conclusion}

In conclusion, we observed that $\left[{ }^{51} \mathrm{Cr}\right]$-EDTA does not pass to the CNS in a major way. However, $\left[{ }^{51} \mathrm{Cr}\right]$-EDTA readily passed the TG by $>30$ times compared to the CNS. Application of CFA or IS (studied at 2, 24 or $48 \mathrm{~h}$ after the application) did not show altered transfer constant to $\left.{ }^{[51} \mathrm{Cr}\right]$-EDTA. Thus, with these experiments we suggest; (i) dural triggered TG inflammation, with IS or CFA does not change BBB passage, and (ii) the TG is readily exposed to circulating molecules and could provide a site where antimigraine drugs could interact with the trigeminal system.

\section{Additional file}

Additional file 1: Figure S1. Analysis of tissue water content. Tissue water content in the investigated tissue structure of the brain and in the trigeminal ganglia is shown. Tissue water content following application of CFA or IS to the dura was compared to brain water content following application of vehicle to the dura. ${ }^{*} p \leq 0.05$. (TIFF $515 \mathrm{~kb}$ ) 


\section{Abbreviations}

BBB: Blood - Brain Barrier; CFA: Complete Freund's Adjuvant; CGRP: Calcitonin Gene-Related Peptide; CNS: Central Nervous System; EBA: Evans Blue Albumin; IL: InterLeukin; IS: Inflammatory soup; MAP: Mean Arterial blood Pressure; MMA: Middle Meningeal artery; PAG: PeriAqueductal Gray; PS: Permeability Surface area product; TG: Trigeminal Ganglion; TNC: Trigeminal Nucleus Caudalis.

\section{Competing interests}

The authors $\mathrm{CL}, \mathrm{KAH}$ and $\mathrm{GG}$ declare no conflict of interest. LE has consulted for TEVA Pharma and Eli Lilly Pharma.

\section{Authors' contributions}

$\mathrm{CL}$ performed the Tissue Water Content and Permeability surface experiments combined with analysis and helped to draft the manuscript. $\mathrm{KAH}$ performed the Evans blue and Photo microscopy and drafted the manuscript. GG contributed in the experiments and analysis. LE conceived of the study, and participated in its design and coordination and helped to draft the manuscript. All authors read and approved the final manuscript.

\section{Funding}

This work was supported by the Lundbeck foundation; Lundbeck Grant of excellence, the heart and lung foundation, the Swedish Research Council, Region Skåne (ALF) and the Medical Faculty of Lund University, Sweden. The funders had no role in study design, data collection and analysis, decision to publish, or preparation of the manuscript.

\section{Received: 2 September 2015 Accepted: 21 October 2015}

\section{Published online: 28 October 2015}

\section{References}

1. Smitherman TA, Burch R, Sheikh H, Loder E (2013) The prevalence, impact, and treatment of migraine and severe headaches in the United States: a review of statistics from national surveillance studies. Headache 3:427-436

2. Akerman S, Holland PR, Goadsby PJ (2011) Diencephalic and brainstem mechanisms in migraine. Nat Rev Neurosci 12:570-584

3. Olesen J, Tfelt-Hansen P, Ashina M (2009) Finding new drug targets for the treatment of migraine attacks. Cephalalgia 29:909-920

4. Ho TW, Edvinsson L, Goadsby PJ (2010) CGRP and its receptors provide new insights into migraine pathophysiology. Nat Rev Neurol 6:573-582

5. Bigal ME, Walter S (2014) Monoclonal antibodies for migraine: preventing calcitonin gene-related peptide activity. CNS Drugs 28:389-399

6. Edvinsson L, Linde M (2010) New drugs in migraine treatment and prophylaxis: telcagepant and topiramate. Lancet 376:645-655

7. Ferrari MD, Roon Kl, Lipton RB, Goadsby PJ (2001) Oral triptans (serotonin 5$\mathrm{HT}(1 \mathrm{~B} / 1 \mathrm{D})$ agonists) in acute migraine treatment: a meta-analysis of 53 trials. Lancet 358:1668-1675

8. Edvinsson L, Tfelt-Hansen P (2008) The blood-brain barrier in migraine treatment. Cephalalgia 28:1245-1258

9. Edvinsson $L$ (2015) CGRP receptor antagonists and antibodies against CGRP and its receptor in migraine treatment. Br J Clin Pharmacol. 80: 193-199 doi:10.1111/ bcp. 12618

10. Edvinsson L, Ho TW (2010) CGRP receptor antagonism and migraine. Neurotherapeutics 7:164-175

11. Hostetler ED, Joshi AD, Sanabria-Bohorquez S, Fan H, Zeng Z, Purcell M, Gantert L, Riffel K, Williams M, O'Malley S, Miller P, Selnick HG, Gallicchio SN, Bell IM, Salvatore CA, Kane SA, Li CC, Hargreaves RJ, de Groot T, Bormans G, Van Hecken A Derdelinckx I, de Hoon J, Reynders T, Declercq R, De Lepeleire I, Kennedy WP, Blanchard R, Marcantonio EE, Sur C, Cook JJ, Van Laere K, Evelhoch JL (2013) In vivo quantification of calcitonin gene-related peptide receptor occupancy by telcagepant in rhesus monkey and human brain using the positron emission tomography tracer [11C]MK-4232. J Pharmacol Exp Ther 347:478-486

12. Kaube H, Hoskin KL, Goadsby PJ (1993) Inhibition by sumatriptan of central trigeminal neurones only after blood-brain barrier disruption. $\mathrm{Br} J$ Pharmacol 109:788-792

13. Bauer HC, Krizbai IA, Bauer H, Traweger A (2014) "You Shall Not Pass"-tight junctions of the blood brain barrier. Front Neurosci 8:392

14. Chodobski A, Ghersi-Egea JF, Nicholson C, Nagaraja TN, SzmydyngerChodobska J (2015) The quest for a better insight into physiology of fluids and barriers of the brain: the exemplary career of Joseph D. Fenstermacher. Fluids Barriers CNS 12:1
15. Eftekhari S, Salvatore CA, Johansson S, Chen TB, Zeng Z, Edvinsson L (2015) Localization of CGRP, CGRP receptor, PACAP and glutamate in trigeminal ganglion. Relation to the blood-brain barrier. Brain Res 1600:93-109

16. Olesen J, Burstein R, Ashina M, Tfelt-Hansen P (2009) Origin of pain in migraine: evidence for peripheral sensitisation. Lancet Neurol 8:679-690

17. Burstein R, Yamamura H, Malick A, Strassman AM (1998) Chemical stimulation of the intracranial dura induces enhanced responses to facial stimulation in brain stem trigeminal neurons. J Neurophysiol 79:964-982

18. Grande G, Labruijere S, Haanes KA, MaassenVanDenBrink A, Edvinsson L (2014) Comparison of the vasodilator responses of isolated human and rat middle meningeal arteries to migraine related compounds. J Headache Pain 15:22

19. Gupta S, Mehrotra S, Avezaat CJ, Villalon CM, Saxena PR, MaassenVanDenBrink A (2006) Characterisation of CGRP receptors in the human isolated middle meningeal artery. Life Sci 79:265-271

20. Ho TW, Olesen J, Dodick DW, Kost J, Lines C, Ferrari MD (2011) Antimigraine efficacy of telcagepant based on patient's historical triptan response. Headache 51:64-72

21. Liu Y, Broman J, Edvinsson L (2008) Central projections of the sensory innervation of the rat middle meningeal artery. Brain Res 1208:103-110

22. Kuris A, Xu CB, Zhou MF, Tajti J, Uddman R, Edvinsson L (2007) Enhanced expression of CGRP in rat trigeminal ganglion neurons during cell and organ culture. Brain Res 1173:6-13

23. Tajti J, Kuris A, Vecsei $L, X u C B$, Edvinsson $L$ (2011) Organ culture of the trigeminal ganglion induces enhanced expression of calcitonin gene-related peptide via activation of extracellular signal-regulated protein kinase 1/2. Cephalalgia 31:95-105

24. Lukacs M, Haanes KA, Zs M, Tajti J, Vecsei L, Warfvinge K, Edvinsson L (2015) Dural administration of inflammatory soup or Complete Freund's Adjuvant induces activation and inflammatory response in the rat trigeminal ganglion. J Headache Pain 16:564

25. Gursoy-Ozdemir Y, Qiu J, Matsuoka N, Bolay H, Bermpohl D, Jin H, Wang X Rosenberg GA, Lo EH, Moskowitz MA (2004) Cortical spreading depression activates and upregulates MMP-9. J Clin Invest 113:1447-1455

26. Cutrer FM, Sorensen AG, Weisskoff RM, Ostergaard L, Del Sanchez RM, Lee EJ, Rosen BR, Moskowitz MA (1998) Perfusion-weighted imaging defects during spontaneous migrainous aura. Ann Neurol 43:25-31

27. Del Sanchez RM, Bakker D, Wu O, Agosti R, Mitsikostas DD, Ostergaard L, Wells WA, Rosen BR, Sorensen G, Moskowitz MA, Cutrer FM (1999) Perfusion weighted imaging during migraine: spontaneous visual aura and headache. Cephalalgia 19:701-707

28. Jager HR, Giffin NJ, Goadsby PJ (2005) Diffusion- and perfusion-weighted MR imaging in persistent migrainous visual disturbances. Cephalalgia 25:323-332

29. Strassman AM, Raymond SA, Burstein R (1996) Sensitization of meningeal sensory neurons and the origin of headaches. Nature 384:560-564

30. Jungner M, Lundblad C, Bentzer P (2013) Rosuvastatin in experimental brain trauma: improved capillary patency but no effect on edema or cerebral blood flow. Microvasc Res 88:48-55

31. Lundblad C, Grande PO, Bentzer P (2008) Increased cortical cell loss and prolonged hemodynamic depression after traumatic brain injury in mice lacking the IP receptor for prostacyclin. J Cereb Blood Flow Metab 28:367-376

32. Fenstermacher JD (1984) Volume regulation of the central nervous system In: Staub NC, Taylor AE (eds) Edema. Raven, New York, pp 383-404

33. Fenstermacher JD, Blasberg RG, Patlak CS (1981) Methods for Quantifying the transport of drugs across brain barrier systems. Pharmacol Ther 14:217-248

34. Blasberg RG, Patlak CS, Fenstermacher JD (1983) Selection of experimental conditions for the accurate determination of blood-brain transfer constants from single-time experiments: a theoretical analysis. J Cereb Blood Flow Metab 3:215-225

35. Lundberg N, West KA (1965) Leakage as a source of error in measurement of the cerebrospinal fluid pressure by lumbar puncture. Acta Neurol Scand Suppl 13(Pt 1):115-121

36. Hoffmann J, Wecker S, Neeb L, Dirnagl U, Reuter U (2012) Primary trigeminal afferents are the main source for stimulus-induced CGRP release into jugular vein blood and CSF. Cephalalgia 32:659-667

37. Burstein $\mathrm{R}$, Jakubowski M (2004) Analgesic triptan action in an animal mode of intracranial pain: a race against the development of central sensitization. Ann Neurol 55:27-36

38. Rosenberg GA, Kornfeld M, Estrada E, Kelley RO, Liotta LA, Stetler-Stevenson WG (1992) TIMP-2 reduces proteolytic opening of blood-brain barrier by type IV collagenase. Brain Res 576:203-207 
39. Markowitz S, Saito K, Moskowitz MA (1987) Neurogenically mediated leakage of plasma protein occurs from blood vessels in dura mater but not brain. J Neurosci 7:4129-4136

40. Williamson DJ, Hargreaves RJ, Hill RG, Shepheard SL (1997) Intravital microscope studies on the effects of neurokinin agonists and calcitonin gene-related peptide on dural vessel diameter in the anaesthetized rat. Cephalalgia 17:518-524

41. Kurosawa M, Messlinger K, Pawlak M, Schmidt RF (1995) Increase of meningeal blood flow after electrical stimulation of rat dura mater encephali: mediation by calcitonin gene-related peptide. Br J Pharmacol 114:1397-1402

Submit your manuscript to a SpringerOpen ${ }^{\odot}$ journal and benefit from:

- Convenient online submission

- Rigorous peer review

- Immediate publication on acceptance

- Open access: articles freely available online

- High visibility within the field

- Retaining the copyright to your article

Submit your next manuscript at $\gg$ springeropen.com 